\title{
Image Analysis Based Estimates of Regolith Erosion Due to Plume Impingement Effects
}

\author{
J.E. Lane ${ }^{1}$ and P.T. Metzger ${ }^{2}$ \\ ${ }^{1}$ Enterprise Advisory Services, Inc., ESC-58 \\ Kennedy Space Center, FL 38299, john.e.lane@nasa.gov \\ ${ }^{2}$ NASA, Granular Mechanics and Regolith Operations Lab, NE-S \\ Kennedy Space Center, FL 38299, phillip.t.metzger@nasa.gov
}

\section{Introduction}

Characterizing dust plumes on the moon's surface during a rocket landing is imperative to the success of future operations on the moon or any other celestial body with a dusty or soil surface (including cold surfaces covered by frozen gas ice crystals, such as the moons of the outer planets). The most practical method of characterizing the dust clouds is to analyze video or still camera images of the dust illuminated by the sun or on-board light sources (such as lasers). The method described below was used to characterize the dust plumes from the Apollo 12 landing.

In this context, the histogram matching method (HMM) is an image processing technique for determining dust optical density in Apollo landing videos. The software implementation of HMM creates a greyscale image histogram and calculates the histogram mean and standard deviation, which is then used to match dusty and clear images for the purpose of estimating an effective optical density and optical extinction coefficient $\alpha$. A dust thickness model, based on the tilt of the camera and increasing height of the dust layer towards the top of the image, is used to account for the distance light travels through the dust.

Previous methods relied on comparing specific features in clear vs. dusty images, which severely limited ability to analyze video frames. This method compares the statistical nature of a clear image to the statistical nature of a dusty image, assuming that the average scene's description (as characterized by an image histogram) due to surface reflectance and sun angle is invariant throughout the frame sequence. This assumption fails when shadows show up on the scene, which is evident in the last $20 \mathrm{~s}$ of the landing descent. In the last $20 \mathrm{~s}$, the error minimization of the histogram matching is by-passed and the matching is done manually by visually comparing images.

Regolith erosion rate and total mass eroded due to a vehicle landing on the lunar surface can be computed by a technique similar to that used by the National Weather Service for estimating rainfall rate from radar reflectivity [1]. With this proposed method, soil erosion rate is similar to rainfall deposition rate, while optical extinction [2] is similar to weather radar reflectivity. Radar meteorology relies on a predetermined average rainfall drop size distribution in order to extract rainfall rate from radar measurements. Similarly, the surface regolith erosion rate due to plume impingement of a landing vehicle [3] depends on knowledge of the soil's particle size distribution [4]. In the case of Apollo landers, optical extinction can be estimated by image analysis of the cockpit camera landing videos. Using this approach, the computed plume induced soil erosion rate is: 


$$
\dot{m}(t)=\frac{\rho_{L} \pi}{3} \cdot \frac{\int_{0}^{\infty} S(D) D^{3} d D}{\int_{0}^{\infty} S(D)\left(D^{2} / v(t, D)\right) d D} \cdot M_{2}(t),
$$

where $\rho_{L}$ is the bulk density of lunar soil (3100 $\left[\mathrm{kg} \mathrm{m}^{-3}\right]$ ). The second moment $M_{2}$ is related to the measured optical extinction by, $\alpha=(\pi / 4) Q_{e} M_{2}$, where $Q_{e}$ is the scattering efficiency factor for extinction [5]. The velocity term, $v(t, D)$ is determined from computation fluid dynamics (CFD) analysis of the Apollo LM descent engine, as well as particle trajectory analysis based on the CFD simulations [6]. See Appendix A for details on modeling $v(t, D) . S(D)$ is the normalized soil size distribution of Apollo soil samples returned from the lunar surface, as measured by a Fine Particle Analyzer, sieves, or something equivalent (see Appendix B). An empirical relationship is established between shear stress as determined by CFD simulation and erosion rate estimated by optical extinction measurements for the case of the Apollo 12 LM.

\section{Histogram Matching Method}

The output of the HMM algorithm is a modified image, where "dust" has been added (mode 1) or removed (mode 0 ). The output image pixel $p_{i j}^{\prime}$ is computed from the input image pixel $p_{i j}$ :

$$
p_{i j}^{\prime}=\left\{\begin{array}{ll}
p_{i j} e^{-2 \alpha x}+p_{0}\left(1-e^{-\alpha x}\right) & \text { mode }=1 \\
p_{0} e^{\alpha x}-\left(p_{0}-p_{i j}\right) e^{-2 \alpha x} & \text { mode }=0
\end{array} .\right.
$$

where $\alpha$ is the optical extinction coefficient of the dust and $p_{0}$ is a fitting parameter associated with the dynamic range of the image (ideally $p_{0}=255$ for an 8-bit image). The factor of two in the extinction term is the result of light reflecting off of the lunar surface back to the camera. The exponent term without the factor of two corresponds to light scattered back to the camera from the dust cloud. The distance $x$ in Equation (2) is the effective optical-dust path length model along the camera view ray through the dust cloud, corresponding to each $i j$ image pixel in the image. It is equal to the physical path length $x_{D}$ of the dust for $r \leq a_{0}$, where $r$ is the radial distance from the engine nozzle centerline and $a_{0}$ is a parameter. For $r>a_{0}$, the effective path length is $x_{D}$ scaled by the radial dispersion factor:

$$
\begin{gathered}
x=\left\{\begin{array}{cc}
x_{D} & r \leq a_{0} \\
x_{D}\left(\frac{a_{0}}{r}\right)^{2} r>a_{0}
\end{array},\right. \\
x_{D}=\frac{\tan \theta \cot \phi\left(l_{c}+h \tan \phi\right) \sec \left(\phi+\tan ^{-1}(q d / F)\right)}{\cot \left(\phi+\tan ^{-1}(q d / F)\right)+\tan \theta},
\end{gathered}
$$

where $F$ is the focal length of the camera $(F=10 \mathrm{~mm}), d$ is the pixel width $(d \approx 15 \mu \mathrm{m}), \theta$ is the dust angle relative to horizontal $\left(\theta \approx 3^{\circ}\right), \phi$ is the camera angle relative to vertical $\left(\phi=33^{\circ}\right), l_{c}=$ $1.2 \mathrm{~m}$ is the camera offset distance from the nozzle center line, and $h$ is height of the LM above 
the surface. The variable $q$ is the vertical distance in the image in pixel units from the ij pixel to a horizontal centerline in the rotated camera view:

$$
q=\left(j-\frac{1}{2} N\right) \cos \xi-\left(i-\frac{1}{2} M\right) \sin \xi \quad,
$$

where $\xi$ is the camera rotation angle about the camera axis $\left(\xi \approx-33^{\circ}\right), i$ is the horizontal pixel index, $j$ is the vertical pixel index, $N$ is the total number of horizontal pixels, and $M$ is the total number of vertical pixels.

The left side of Figure 1 shows frame F3077 $(h=34 \mathrm{~m}$ ) of the cockpit video camera. The right side of Figure 1 displays a map of the same field of view for this frame, showing the effective optical-dust path length model, Equation (3), which is based on the tilt of the camera and increasing depth of the dust layer towards the top of the image. The horizontal and vertical axes of the plot are in pixel units. The contours are graded in increments of $0.3 \mathrm{~m}$, starting with the minimum, $x=0.3 \mathrm{~m}$ at the bottom (purple in the on-line version), to a maximum, $x=3.0 \mathrm{~m}$ at the top (red in the on-line version).

Figure 2 is a similar image set, occurring $46.6 \mathrm{~s}$ later at an LM altitude of $h=11 \mathrm{~m}$. The contours are graded in increments of $0.017 \mathrm{~m}$, starting with the minimum, $x=0.1 \mathrm{~m}$ at the bottom (purple in the on-line version), to a maximum, $x=0.25 \mathrm{~m}$ near the center (red in the on-line version). Note that the video frame numbers F3077 (Figure 1) and F3543 (Figure 2) correspond to a constant frame rate of $10 \mathrm{fps}$.

The HMM algorithm processes two input images, pixel by pixel. The first input image is represented by pixel $p_{i j}$ as shown by Equation (2). A reference image is represented by $q_{i j}$. For mode $=0$, The HHM algorithm applies the transformation described by Equation (2) to the input image $p_{i j}$ (frame with dust), creating an output image $p_{i j}^{\prime}$ (artificially removed dust), as shown in Figure 3. The reference image $q_{i j}$ (no dust) is then compared to $p_{i j}^{\prime}$ and by matching the average and standard deviation of the their histograms, the parameters $\alpha, p_{0}$, and $a_{0}$ are found. Figure 4 shows a similar example for mode $=1$.

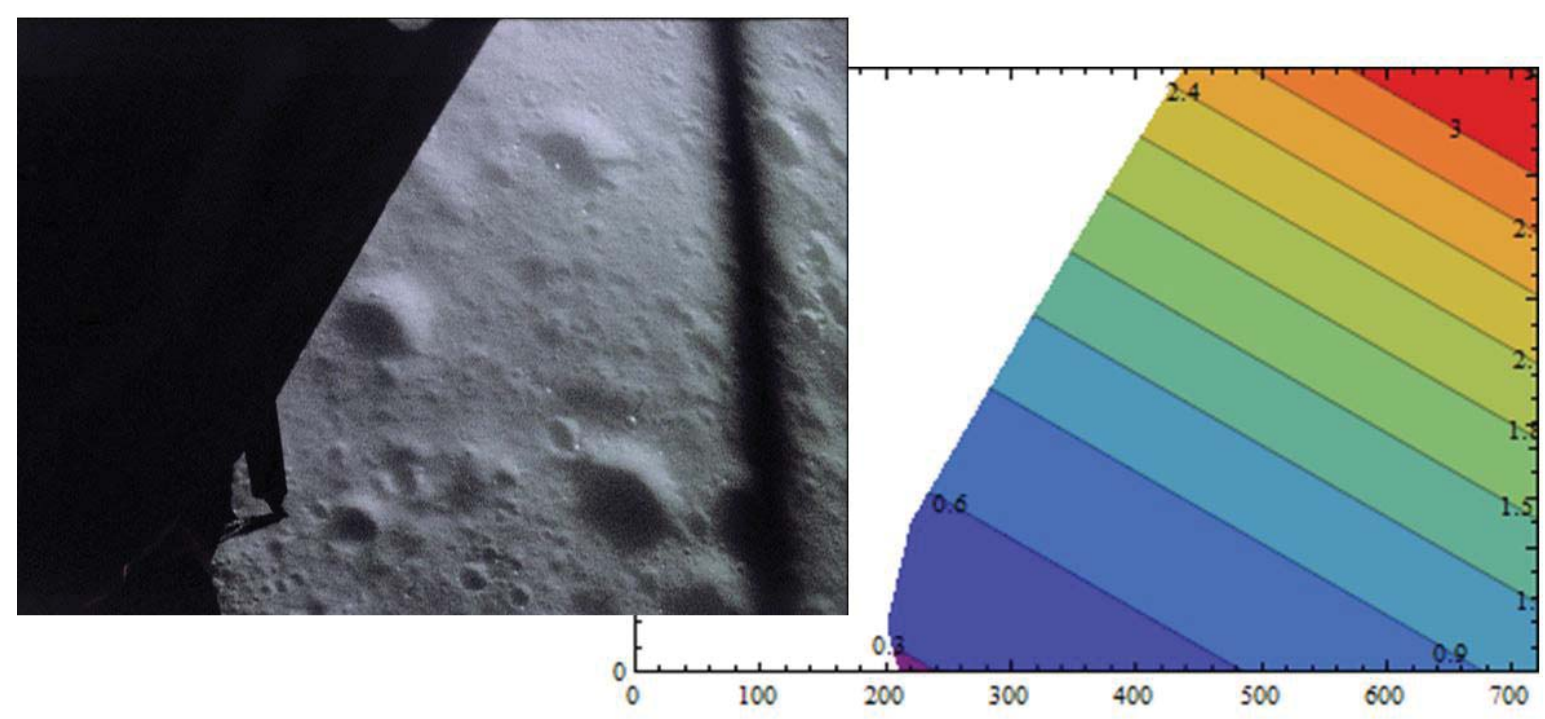


Fig. 1. Dust depth model: (left) video camera frame (F3077), with LM altitude $h=34 \mathrm{~m}$; (right) effective camera dust length $x$ with radius $a_{0}=46 \mathrm{~m}$.

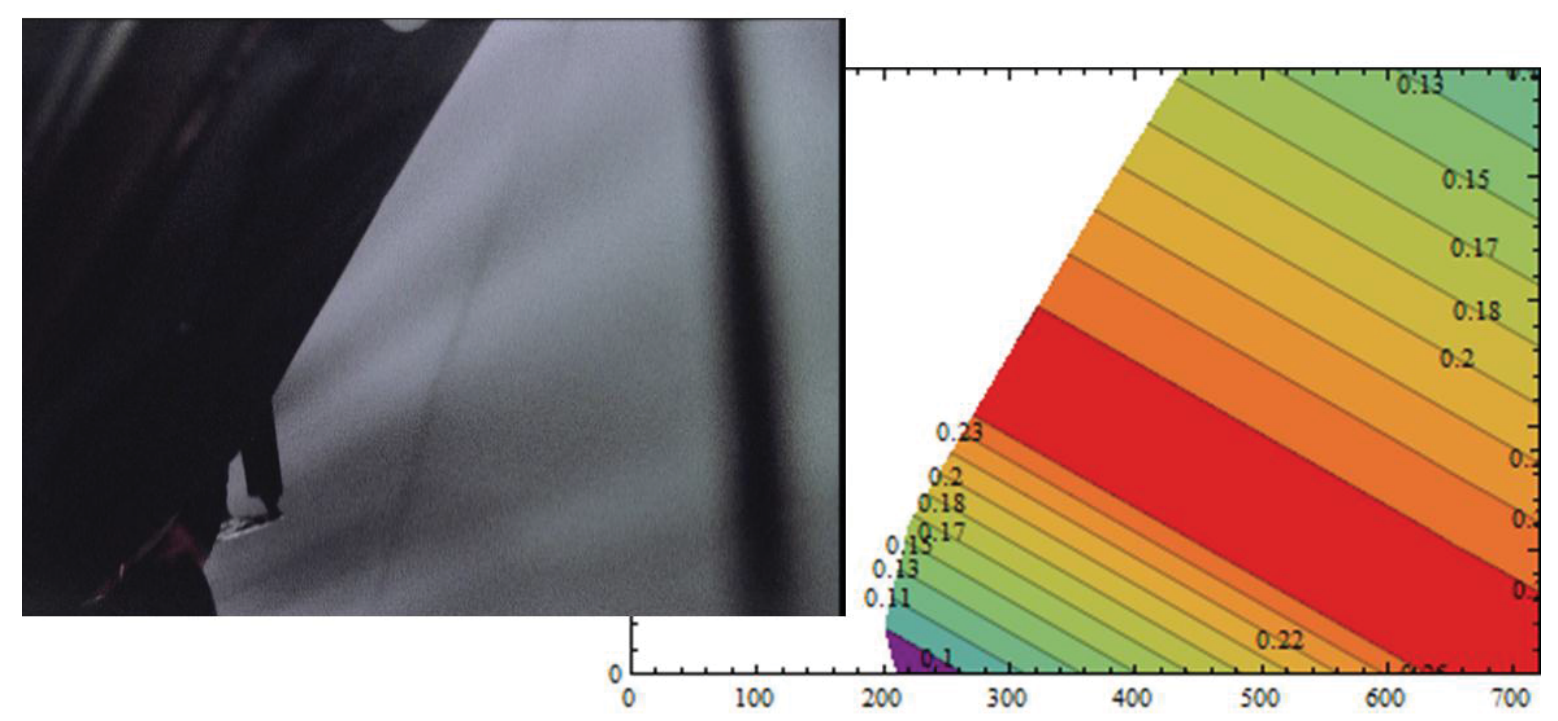

Fig. 2. Dust depth model: (left) video camera frame (F3543), with LM altitude $h=11 \mathrm{~m}$; (right) effective dust depth with radius $a_{0}=6.5 \mathrm{~m}$. 


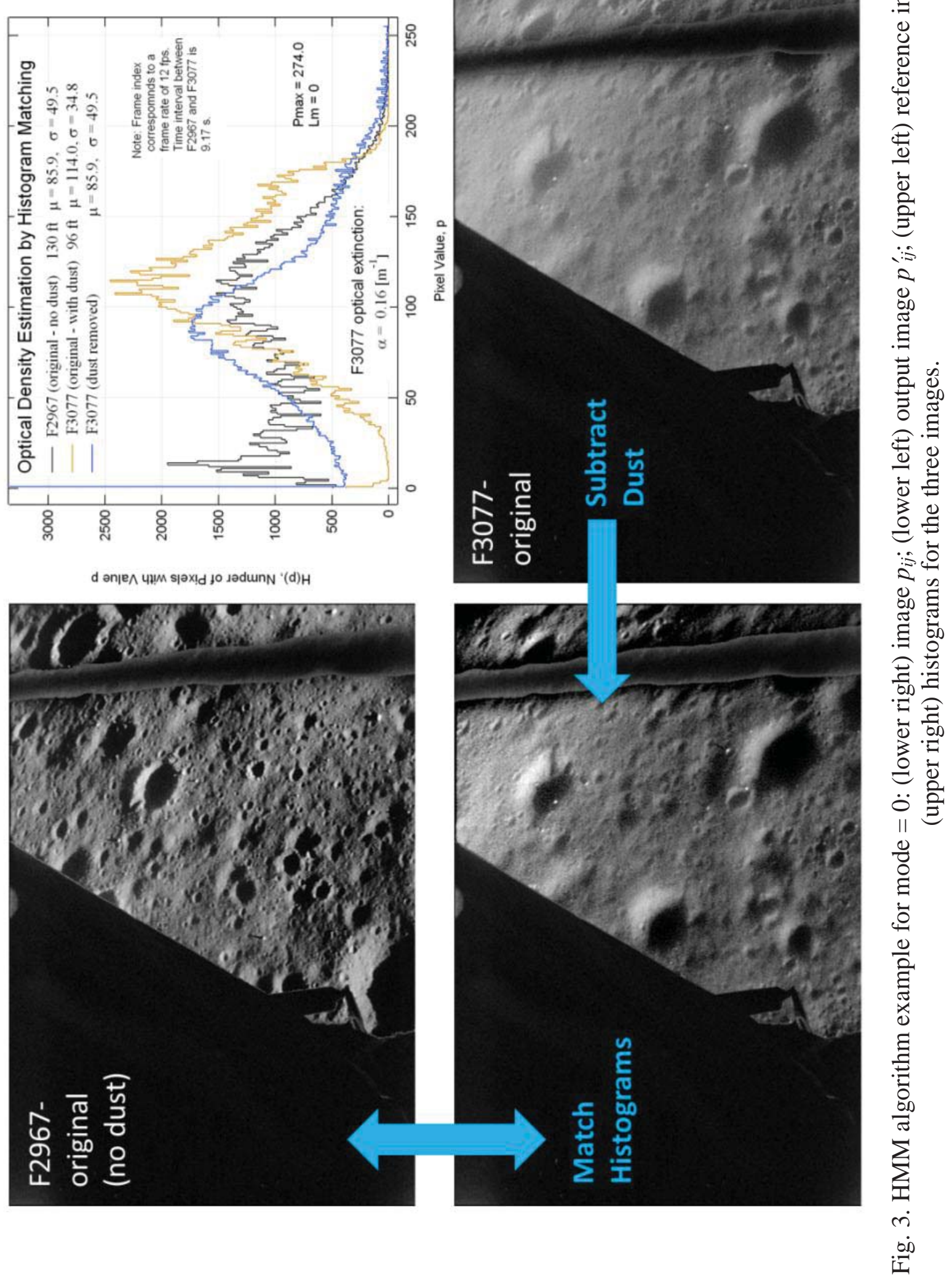




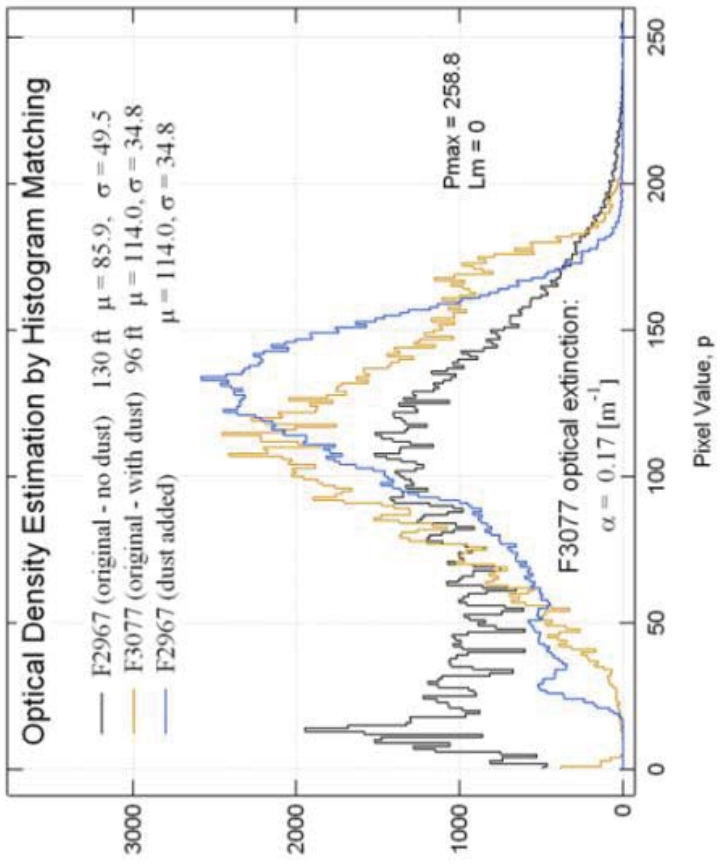

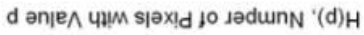

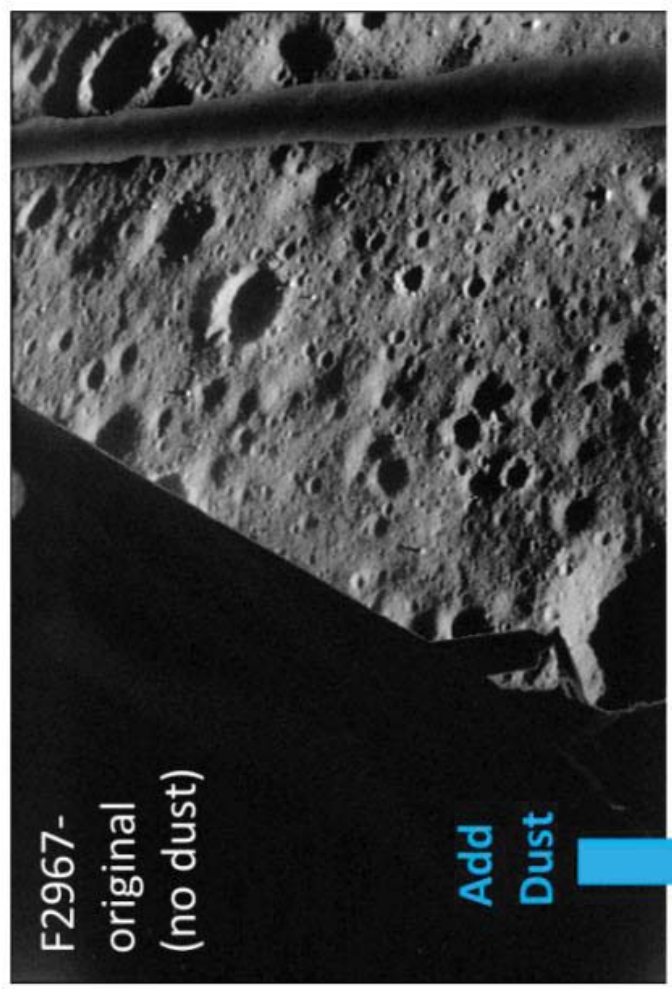

$\stackrel{\square}{\widetilde{\Xi}}$

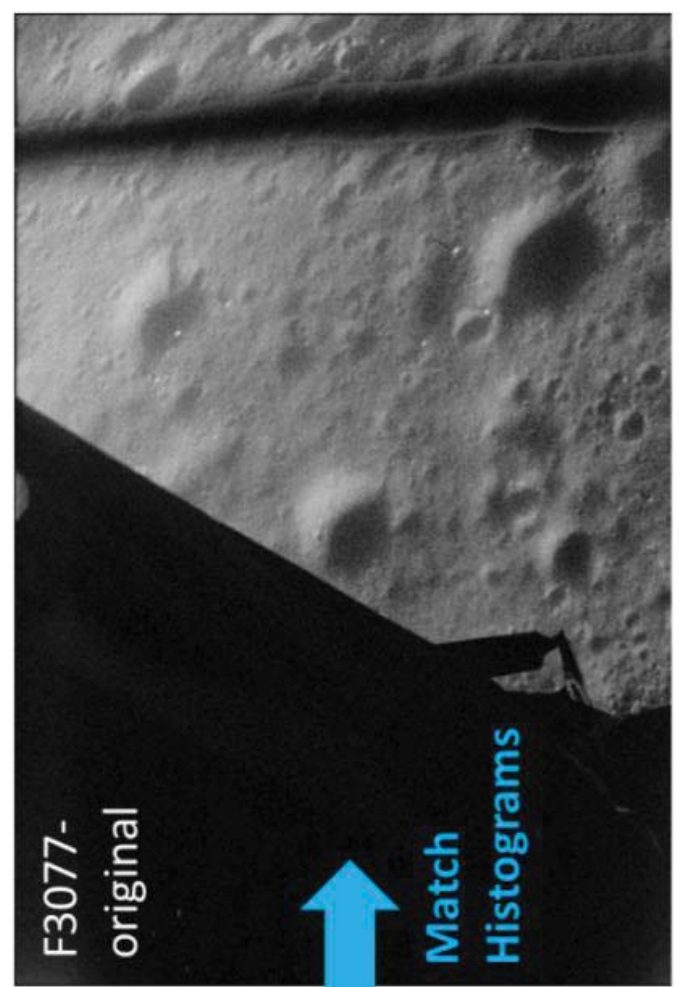

(8)

$\because=$

2

๙ิ

.

节

임

电

它岂

을

:

कू

号营

콜.

录

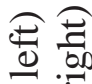

它

刍

$11: \overrightarrow{0}$

.

资

$\stackrel{\infty}{\dot{1}}$ 
The extinction coefficient $\alpha$ can be estimated from the Apollo videos by adding dust to a clear image (before dust appears) and comparing to the dusty image of interest (see Figure 5). Reiterating from the last section, $\alpha=\pi / 4 Q_{e} M_{2}$, where $Q_{e}$ is the scattering efficiency, assumed to be equal to 2. The second moment in Equation (1) is the product of the measured extinction coefficient and $2 / \pi$. The total mass rejected (total mass displaced) is Equation (1) integrated over vehicle descent time and over the area where soil is eroded:

$$
\begin{aligned}
m_{T} & =2 \pi \int_{-\infty}^{0} \int_{0}^{a_{0}(t)} \dot{m}(t) r d r d t \\
& =\pi \int_{-\infty}^{0} a_{0}^{2}(t) \dot{m}(t) d t
\end{aligned}
$$

where $a_{0}(t)$ is the radius on the surface, referenced to the engine nozzle centerline, where erosion is taking place. The assumption inherent in Equation (6) is that erosion is uniform over a circle of radius $a_{0}(t)$ and zero outside of that circle. An estimate of $a_{0}(t)$ for the Apollo $12 \mathrm{LM}$ is shown in Figure 5, which is an output of the HMM algorithm. Note that in this and all previous discussions of $S(D)$ and its moments, it has been assumed that the particle size distribution is homogeneous over the extent of measurement, i.e., within a circle of radius $a_{0}(t)$, and all temporal effects due to engine LM altitude and thrust occur instantaneously over this spatial extent.

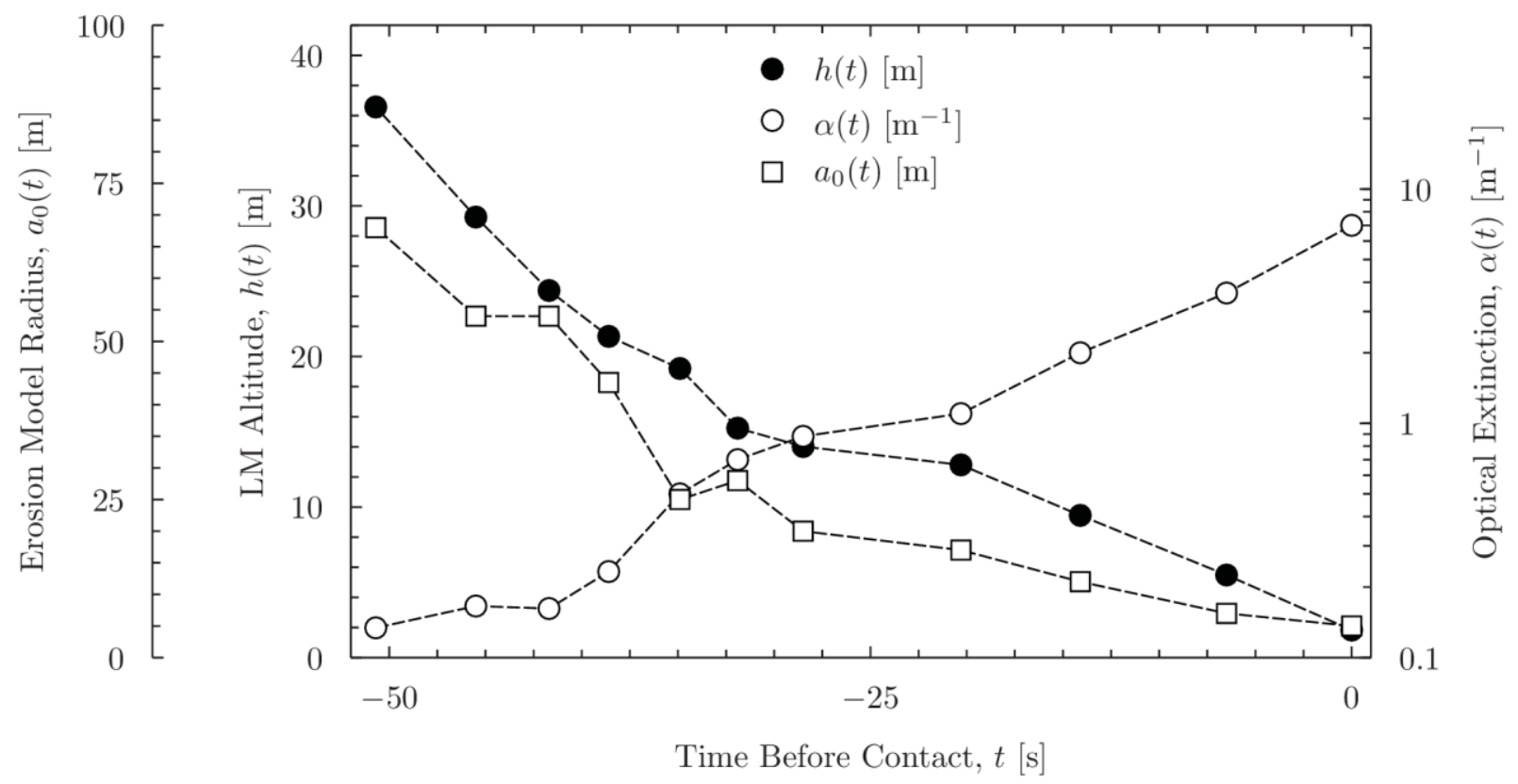

Fig. 5. Apollo 12 optical extinction estimate using histogram matching method. The time intervals correspond to the voice callouts of LM pilot, Alan Bean.

The erosion rate should actually vary with the state of the gas flowing across the soil, including its shear stress, rarefaction, and turbulence; it should also vary with saltation, including the downward flux of larger particles that are too heavy to be carried away by the gas as well as 
smaller particles that are scattered back down from the entrained cloud via particle collisions. Examination of the sandblasting effects on Surveyor III have shown that the downward flux of scattered particles is significant [7] and discrete element computer simulations show the important but largely unexplored role of mid-flight particle scattering in enhancing erosion rate [8]. Influence of the gas upon erosion rate should be greatest in an annular region around the vehicle [9] while the influence of saltation may be greater in another annulus with larger radius since particles travel downrange before striking the surface. Thus the net erosion rate may be somewhat more uniform and spread over a broader region than if gas effects alone are considered. The details of erosion physics -- especially in lunar rocket exhaust conditions -- are not yet well understood, so a constant erosion over a finite area assumed, as it is the simplest model and therefore a sensible first step.

\section{Conclusions}

A method for estimating lunar soil erosion rate due to plume impingement of the Apollo $12 \mathrm{Lu}-$ nar Module Intrepid during its descent to the lunar surface has been presented. The observables are optical extinction and particle size distributions of soil samples returned from the lunar surface. The optical extinction is measured between the camera mounted inside of the cockpit window and the lunar surface during landing. CFD analysis of the Apollo LM descent engine, as well as particle trajectory analysis based on the CFD simulations, provides the remainder of the necessary data.

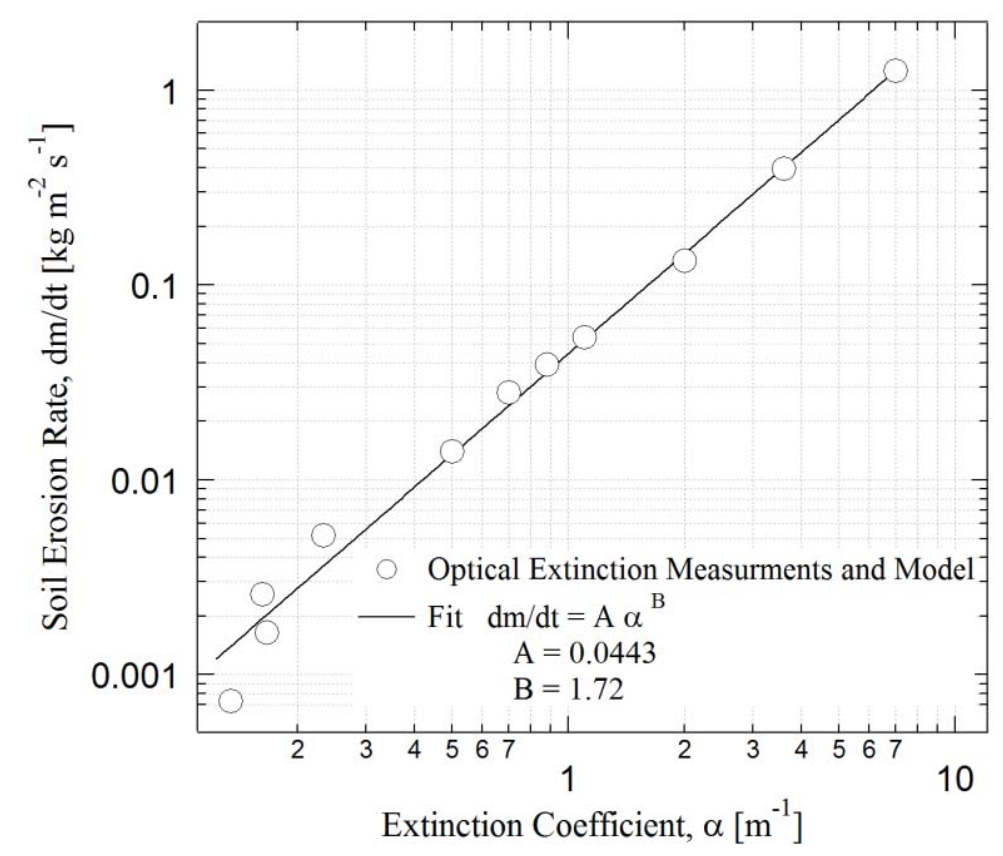

Fig. 6. Soil mass erosion rate $\dot{m}$ versus Optical extinction $\alpha$, showing power law fit.

The CFD simulations provide two key pieces of information: the velocity profile of particles as a function of starting distance from the engine nozzle, size of the particle, and height of the lander from the surface. Taking an approach similar to the problem of estimating rainfall rate from weather radar, Equation (1) was presented as the solution to the problem of estimating soil ero- 
sion rate from optical extinction measurements (see Figure 6). In both cases, the particle velocities must be known, as well as the particle size distributions. Quantifying the particle velocity function is in some sense the most difficult part of the problem for both lunar plume observations and weather radar estimation of rainfall. For this reason, the velocity function is likely the source of greatest error.

The optical extinction model described above assumes from the start that the erosion rate is uniform over an area defined by radius $a_{0}$, which is a function of lander height. The primary influence of optical extinction as measured by a reduction of brightness of the surface and increase in brightness of the dust cloud, is the spatial dust geometry. In this model, erosion is uniform over $\pi a_{0}^{2}$ but diverges radially from all points on the surface within $r \leq a_{0}$. No erosion occurs for $r>$ $a_{0}$. This approach is loosely analogous to methods traditionally employed for estimating rainfall rate from radar, where spatial and temporal averaging strategies are necessary to correlate rainfall accumulation rates and radar reflectivity.

The result of the Apollo 12 shear stress to erosion rate relation shows an approximate 5/2 power dependence of erosion rate on shear stress. Theoretical considerations predict a linear relationship [10]. The difference is either a consequence of effects not considered by the theory, or errors in the measurements.

Acknowledgments. We gratefully acknowledge support from NASA's Lunar Advanced Science and Exploration Research (LASER) program, grant NNH10ZDA001N.

\section{References}

[1] Wexler, R., \& Atlas, D. (1963). Radar reflectivity and attenuation of rain. Journal of Applied Meteorology, 2, 276-280.

[2] Atlas, D. (1953), Optical extinction by rainfall, J. Atmos. Sci. 10, 486-486.

[3] Morris, A. B., Goldstein, D. B., Varghese, P. L., \& Trafton, L. M. (2011, May). Plume Impingement on a Dusty Lunar Surface. In AIP Conference Proceedings (Vol. 1333, p. 1187).

[4] Metzger, P.T., Smith, J. and Lane, J.E. (2011) "Phenomenology of Soil Erosion by Rocket Exhaust on the Moon and the Mauna Kea Lunar Analog Site," JGR - Planets, 116:E06005.

[5] van de Hulst, H. C. (2012). "Light scattering by small particles". Courier Dover Publications.

[6] Lane, J. E., P. T. Metzger, and J. W. Carlson (2010), Lunar dust particles blown by lander engine exhaust in rarefied and compressible flow, paper presented at Earth and Space 2010, 12th Biennial Aerospace Division International Conference on Engineering, Construction and Operations in Challenging Environments, Am. Soc. of Civ. Eng., Honolulu, Hawaii.

[7] Immer, C. D., P. Metzger, P. Hintze, A. Nick, and R. Horan (2011a), Apollo 12 lunar module exhaust plume impingement on lunar Surveyor III, Icarus, 211, 1089-1102, doi:10.1016/j.icarus.2010.11.013.

[8] Berger, K. J., Anand, A., Metzger, P. T., \& Hrenya, C. M. (2013). Role of collisions in erosion of regolith during a lunar landing. Physical Review E, 87(2), 022205.

[9] Roberts, Leonard (1963). "The Action of a Hypersonic Jet on a Dust Layer," IAS Paper No. 63- 50, Institute of Aerospace Sciences 31st Annual Meeting, New York.

[10] Metzger, P. T., E. J. Lane, C. D. Immer, J. N. Gamsky, W. Hauslein, X. Li, R. C. Latta III, and C. M. Donahue (2010), Scaling of erosion rate in subsonic jet experiments and Apollo lunar module landings, paper presented at Earth and Space 2010, 12th Biennial Aerospace Division International Conference on Engineering, Construction and Operations in Challenging Environments, Am. Soc. of Civ. Eng., Honolulu, Hawaii. 


\section{APPENDIX A: Particle Velocity Function}

Particle trajectory modeling, based on CFD simulations of the Apollo LM engine and the lunar environment, yield a particle velocity function which can be described by the empirical fit shown in Equation (A-1):

$$
v(h, D)=\left\{\begin{array}{cc}
0 & \log D+c_{1} \log h>c_{0} \\
\frac{10^{\left(b_{1}+b_{2} \log h\right) \tanh \left(b_{3}+b_{4} \log h\right)}}{b_{0}\left(h^{9 / 20}+D^{1 / 2}\right)} & \text { otherwise }
\end{array},\right.
$$

where $b_{0}=0.2964, b_{1}=-0.225, b_{2}=0.1954, b_{3}=5, b_{4}=4.343, c_{0}=2.212$, and $c_{1}=3.53$. Equation (A-1) is based on the particle trajectory results shown in Figues A-1 through A-3. For every point in $\{h, D\}$ space a distribution of particle velocities is computed from the particle trajectory code using an equivalent Monte Carlo distribution of initial particle trajectory starting points, height above the surface and horizontal distance from the engine nozzle centerline.

Because of the distribution of particle velocities for a given $h$ and $D$, the maximum values originate near the engine nozzle. Since the erosion area within $r<a_{0}$ (where $r$ is the radial distance from the engine nozzle centerline) is much greater for slower velocities, then it reasonable to expect $\eta$ to be much smaller than 1 .

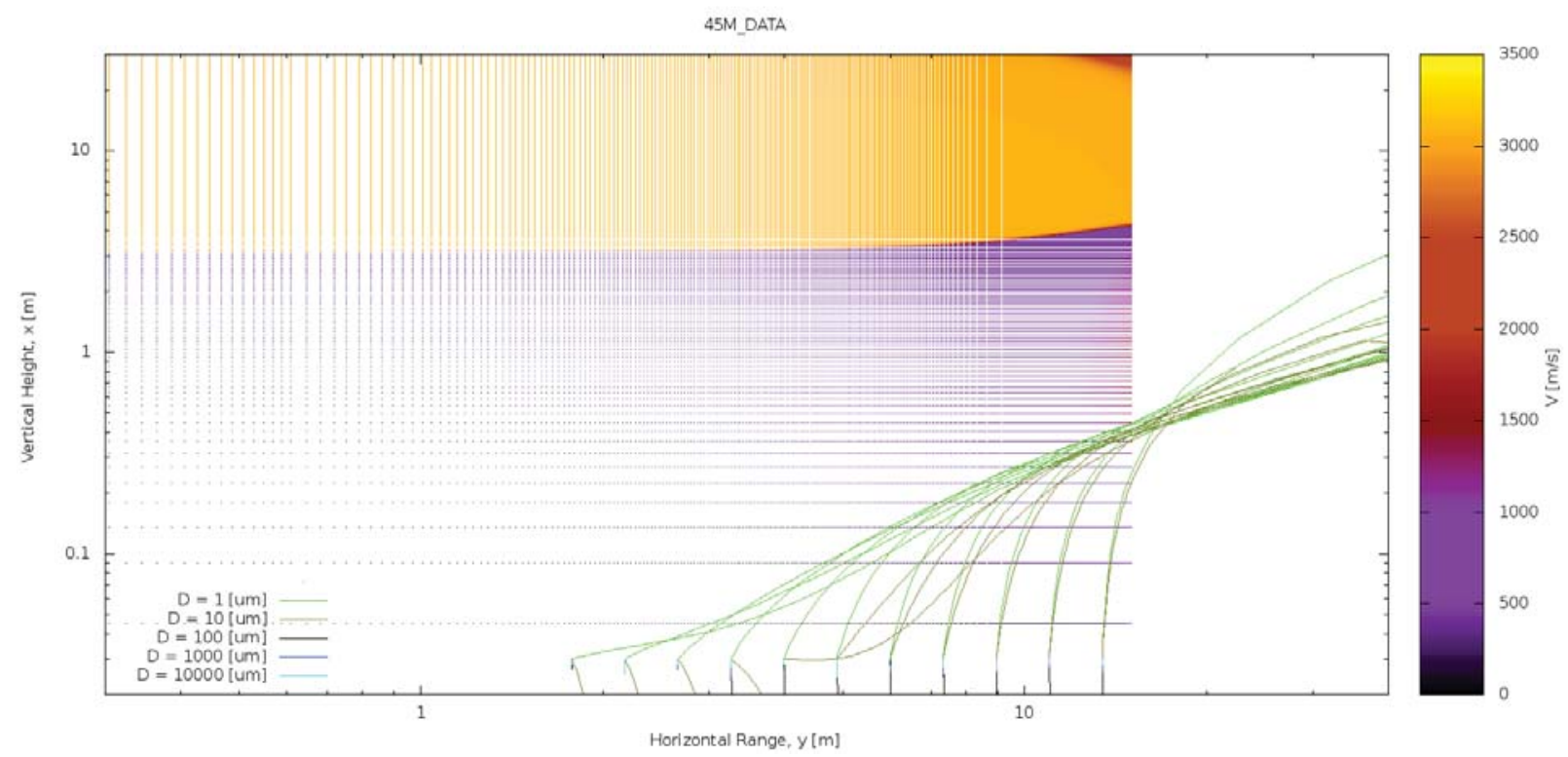


$45 \mathrm{M}(h=45 \mathrm{~m}): x_{0}=0.03 \mathrm{~m}, D=1 \mu \mathrm{m}$

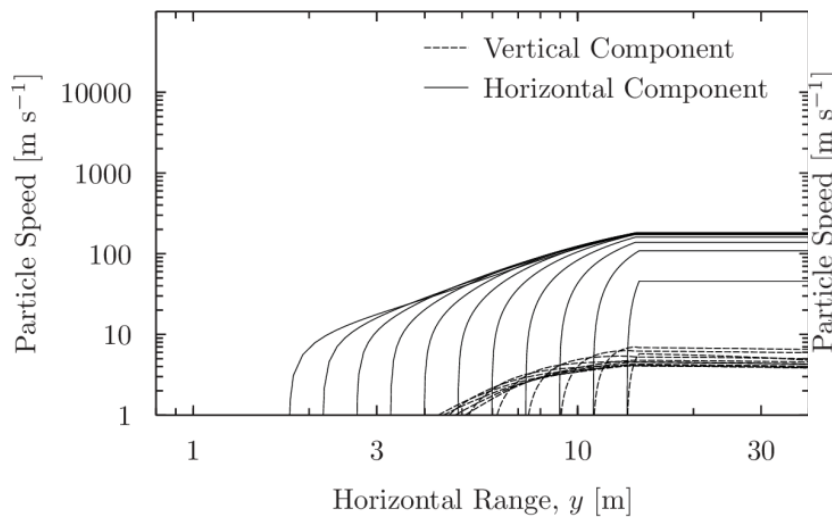

$45 \mathrm{M}(h=45 \mathrm{~m}): x_{0}=0.03 \mathrm{~m}, D=100 \mu \mathrm{m}$

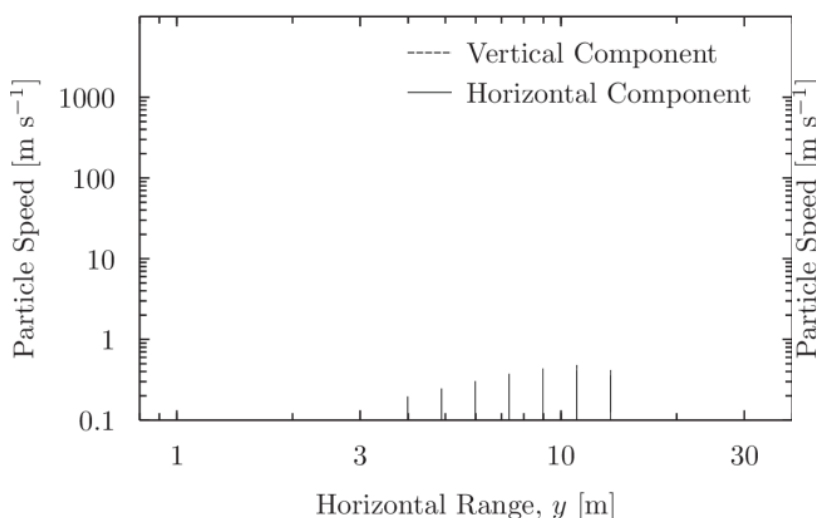

$45 \mathrm{M}(h=45 \mathrm{~m}): x_{0}=0.03 \mathrm{~m}, D=10 \mu \mathrm{m}$

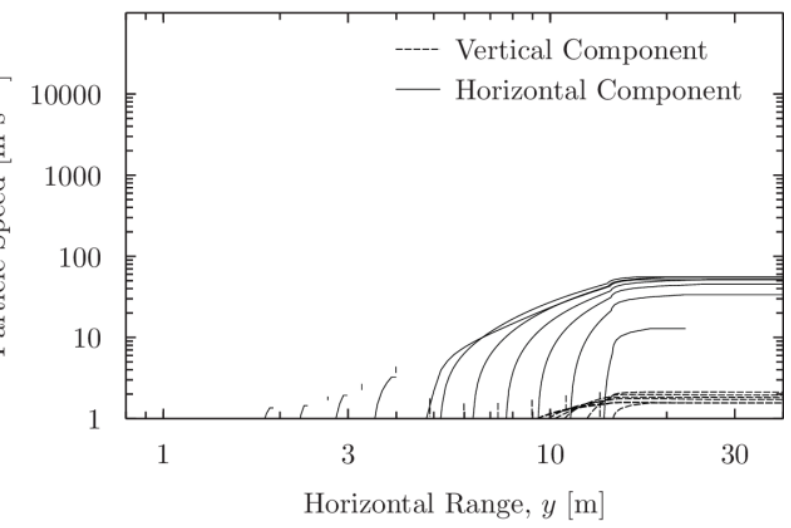

$45 \mathrm{M}(h=45 \mathrm{~m}): x_{0}=0.03 \mathrm{~m}, D=1000 \mu \mathrm{m}$

Fig. A-1. $h=45$ m case:

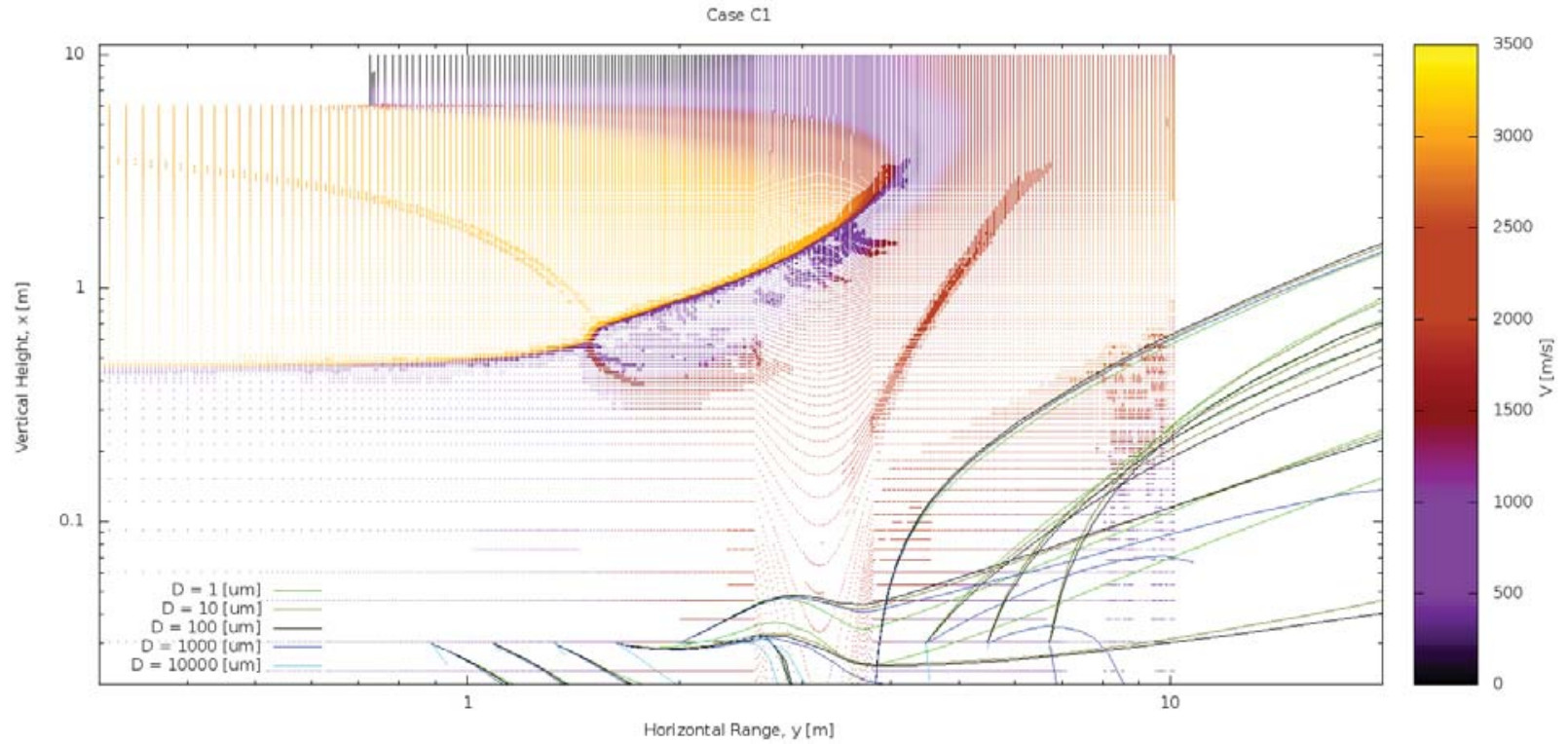


Case C1 $(h=6.1 \mathrm{~m}): x_{0}=0.03 \mathrm{~m}, D=1 \mu \mathrm{m}$

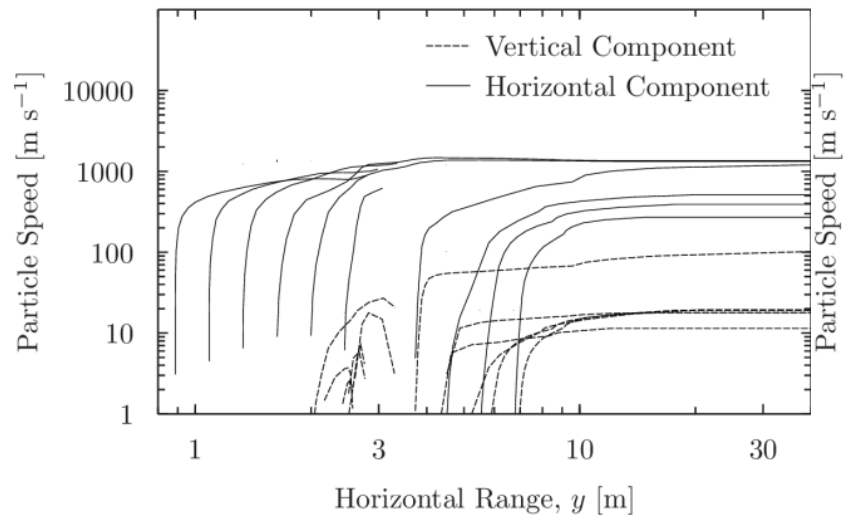

Case C1 $(h=6.1 \mathrm{~m}): x_{0}=0.03 \mathrm{~m}, D=100 \mu \mathrm{m}$

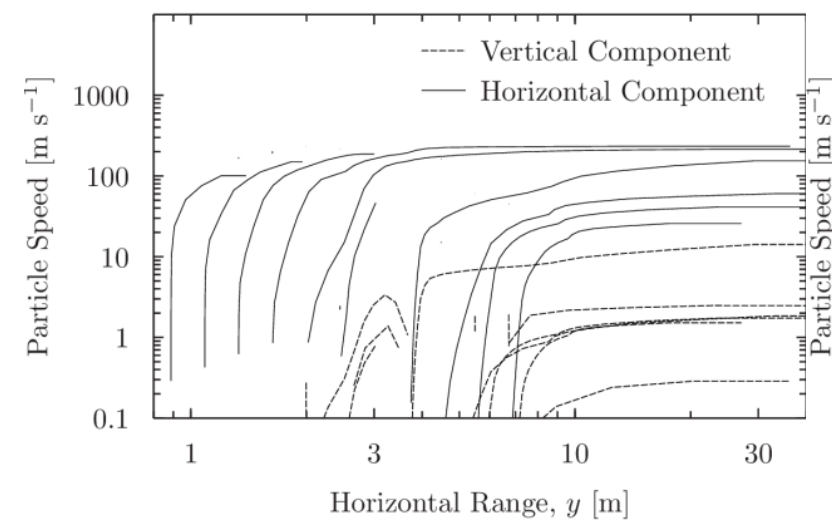

Case C1 $(h=6.1 \mathrm{~m}): x_{0}=0.03 \mathrm{~m}, D=10 \mu \mathrm{m}$

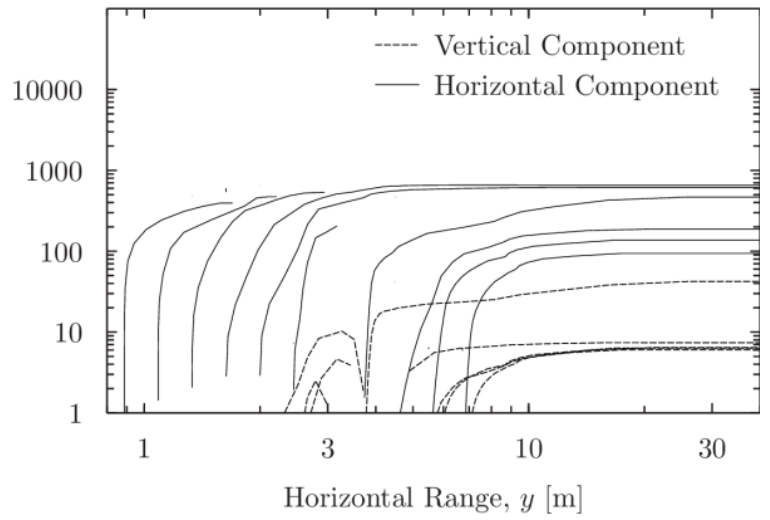

Case C1 $(h=6.1 \mathrm{~m}): x_{0}=0.03 \mathrm{~m}, D=1000 \mu \mathrm{m}$

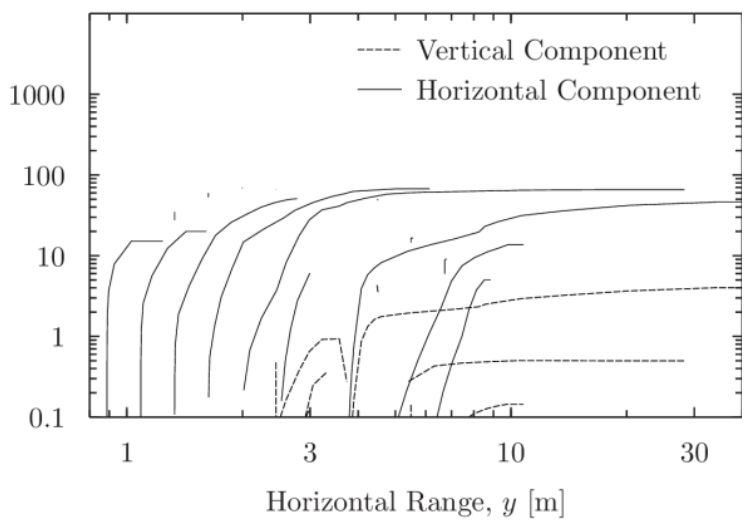

Fig. A-2. $h=6 \mathrm{~m}$ case.

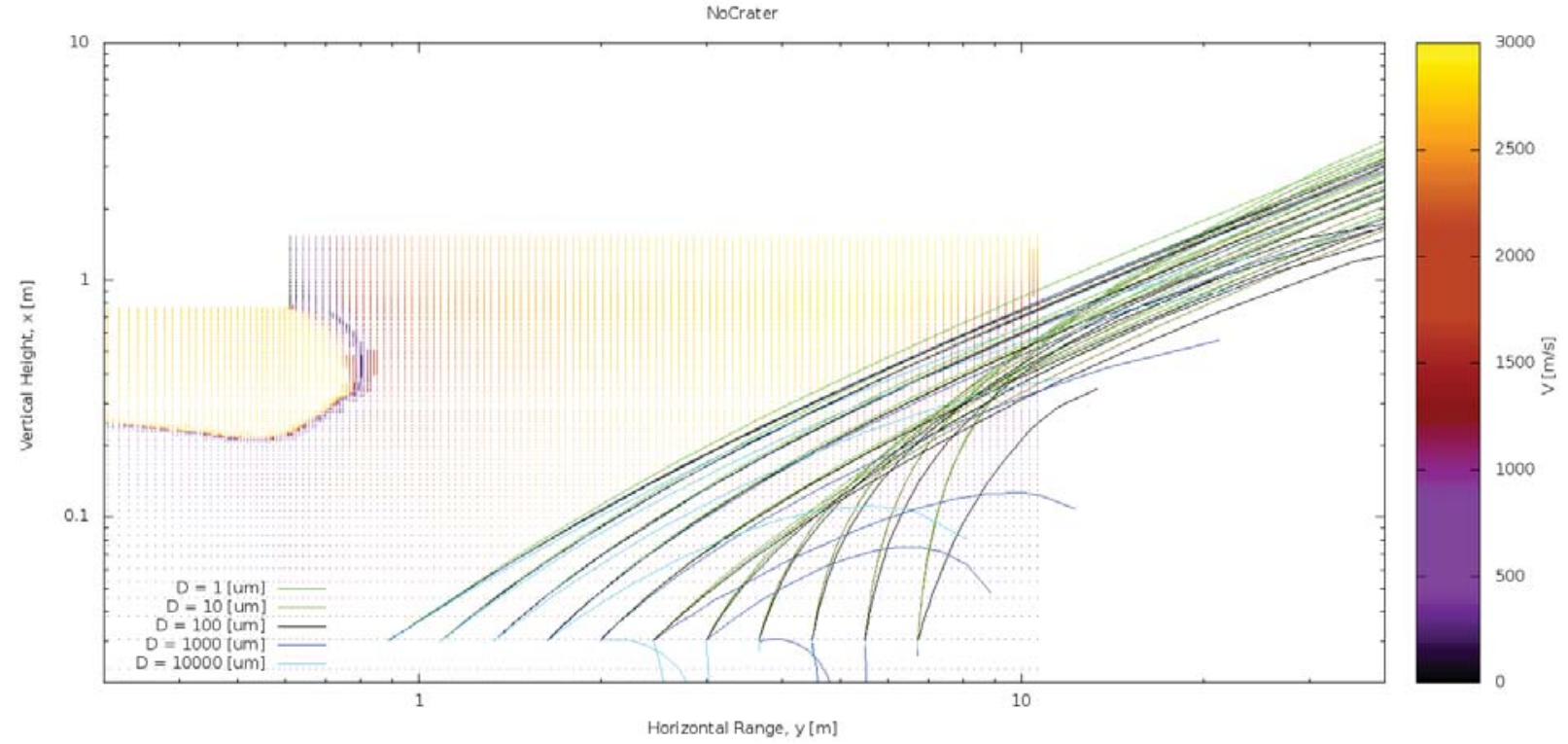



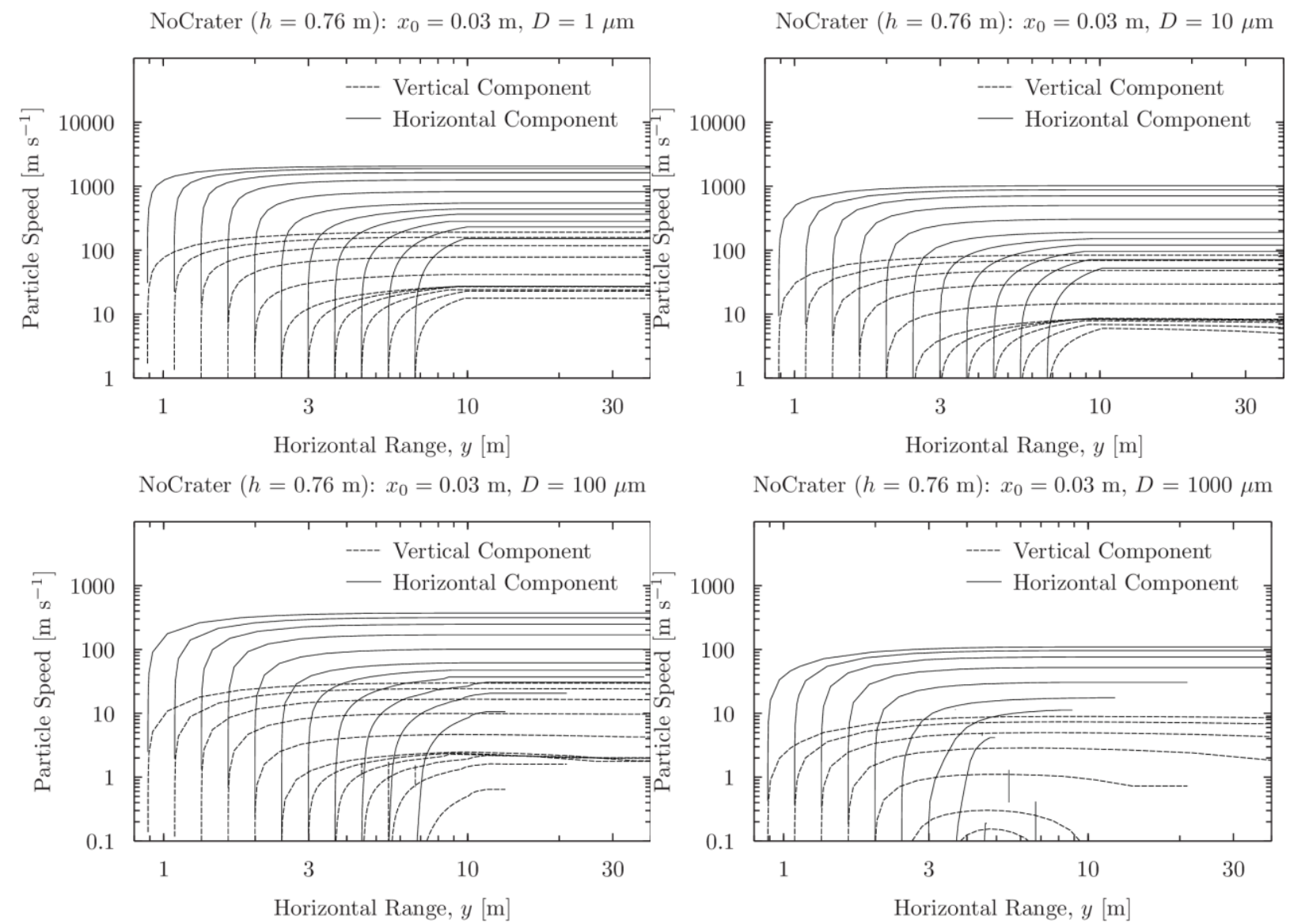

NoCrater $(h=0.76 \mathrm{~m}): x_{0}=0.03 \mathrm{~m}, D=1000 \mu \mathrm{m}$

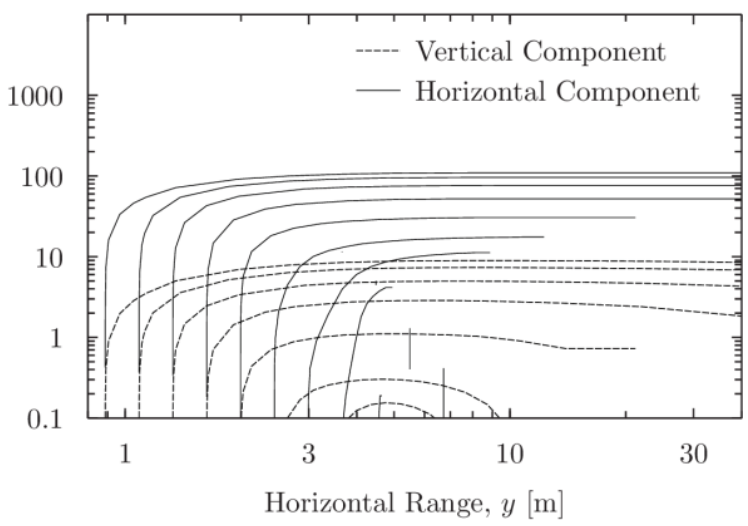

Fig. A-3. $h=1.5 \mathrm{~m}$ case.

\section{APPENDIX B: Particle Size Distribution}

The particle size fraction of the lunar soil at the Apollo 12 site is modeled as a combination of two power law functions by fitting Apollo 11 and 17 soil sample data, as well as JSC-1a simulant [10]:

$$
S(D)=\frac{\left(w(D) S_{1}(D)^{-1}+S_{2}(D)^{-1}\right)^{-1}}{\int_{0}^{\infty}\left(S_{1}(D)^{-1}+w(D) S_{2}(D)^{-1}\right)^{-1} d D}\left[\mathrm{~m}^{-1}\right],
$$

where $S_{1}(D)=\left(D / D_{1}\right)^{-B_{1}}, S_{2}(D)=\left(D / D_{2}\right)^{-B_{2}}$, and $w(D)=\left(D / D_{3}\right)^{-18}+1$. The fitting constants in Equation (B-19) are $D_{1}=4.090 \times 10^{-7}[\mathrm{~m}], B_{1}=1.8, D_{2}=9.507 \times 10^{-6}[\mathrm{~m}], B_{2}=5.6$, and $D_{3}=2.5 \times 10^{-8}$ [m]. The denominator of Equation (B-1) is equal to $4.784 \times 10^{-6}$. Note that all units are kept in meters even though the numbers are more aesthetically pleasing in micrometers. The reason for doing this is to minimize confusion in the integrals involving $S(D)$. 